\title{
SOBRE LA ESTRUCTURA Y LOS LÍMITES DE LOS DERECHOS SOCIALES*
}

\author{
On the structure and limits of social rights
}

\author{
José Manuel Cabra Apalategui ${ }^{1}$ \\ Profesor Contratado Doctor \\ Universidad de Málaga \\ jcabra@uma.es
}

doi: http://dx.doi.org/10.18543/ed-66(1)-2018pp197-213

Recibido: 12.04 .2018

Aceptado: 25.06.2018

\section{Resumen}

Los derechos en general y los derechos sociales en particular se enfrentan a tres tipos de problemas: problemas filosóficos relativos a su fundamentación; problemas políticos que afectan a su reconocimiento; $\mathrm{y}$, por último, problemas jurídicos que surgen a la hora de su garantía y protección. La pregunta acerca del status constitucional de los derechos sociales afecta directamente a este último problema.

En este trabajo voy a mantener dos tesis, que, si bien son conceptualmente independientes, guardan una estrecha relación. En primer lugar, cualquier acercamiento a los problemas jurídicos de los derechos sociales debe tener en cuenta la estructura de los derechos, esto es, la posición del titular y del obligado por el derecho. Tener un derecho, al margen de cuál pueda ser su fundamento, significa ocupar una posición

* Cómo citar / Citation 'Chicago-Deusto' (Autor-fecha / Author-date / Lista de referencias / Reference list entries): Cabra Apalategui, José Manuel. 2018. «Sobre la estructura y los límites de los derechos sociales». Estudios de Deusto 66, n. ${ }^{\circ}$ 1: 197-213. doi: http://dx.doi.org/10.18543/ed-66(1)-2018pp197-213.

${ }^{1}$ Este trabajo se enmarca en el proyecto de investigación «CONFLICTOS DE DERECHOS: TIPOLOGÍAS, RAZONAMIENTOS, DECISIONES» (DER2016-74898-C21-R) del Ministerio de Economía y Competitividad / AEI y FEDER. 
determinada en el marco de una relación jurídica. Afirmar que se tiene derecho a algo sin indicar quién está afectado, como obligado a hacer o a abstenerse de hacer, es una declaración política o moral, pero no el reconocimiento de un derecho en sentido estricto, esto es, en sentido jurídico.

En la segunda parte señalaré que los límites que afectan a la garantía y protección de los derechos sociales dependen de la estructura de los derechos más que de su status constitucional.

\section{Palabras clave}

Derechos sociales; naturaleza relacional de los derechos; estructura de los derechos fundamentales; límites de los derechos.

\section{Abstract}

Rights in general and, particularly, social rights raise problems of three kinds: philosophical problems regarding their foundation; political problems that affect their recognition; and, last, legal problems at the time of their granting and protection. The question on the constitutional status o social rights points out directly to this last problems.

In this paper I am going to defend two thesis that, though conceptually independent, are closely related to each other. First, that any approach to the legal problems of social rights should keep in mind the structure of rights, that is, the position of that entitled and that obligated by the right. To have a right, regardless of its foundation, means to be in a cetain position within a legal relation. To say "I have a right" without identify who is affected by it as the liable party, is just a political or a moral assertion, but not a right strictly speaking, that is, a legal right.

In the second part I will hold that the limits affecting the protection of social rights depend more on the structure of rights than on their constitutional status.

\section{Keywords}

Social Rights; Relational Nature of Rights; Structure of Fundamental Rights; Rights Limits. 
SUMARIO: I. INTRODUCCIÓN. II. UNA TEORÍA ESTRUCTURAL DE LOS DERECHOS FUNDAMENTALES. 1. Enunciados sobre derechos. 2. Las posiciones jurídicas. 2.1. Derechos a algo. 2.2. Libertades. 2.3. Competencias. 3. Los derechos fundamentales como un conjunto de posiciones jurídicas. III. LOS LÍMITES DE LOS DERECHOS. 1. Límites epistémicos. 2. Límites económicos. 3. Límites conceptuales. 4. Límites políticos. BIBLIOGRAFÍA.

\section{INTRODUCCIÓN}

Hace más de cincuenta años, Norberto Bobbio sostuvo que, una vez suscrita por la mayoría de los Estados la Declaración Universal de los Derechos Humanos, el problema de fondo de los derechos no era el de su justificación, sino el de su protección. El consenso generalizado en torno a los derechos había desplazado el foco de atención y el problema no era ya filosófico, sino político, en el bien entendido de que no se está hablando aquí únicamente de voluntad política, sino de que cualquier investigación sobre los fundamentos de los derechos carecerá de importancia si no analiza igualmente las condiciones y los medios para la realización de los distintos derechos ${ }^{2}$.

Así, los derechos en general y los derechos sociales en particular se enfrentan a tres tipos de problemas: problemas filosóficos relativos a su fundamentación; problemas políticos que afectan a su reconocimiento; y problemas jurídicos que surgen a la hora de protegerlos y garantizarlos. Superados los problemas de fundamentación - de hecho, algunos de estos derechos están ya incluidos en la Declaración Universal y son desarrollados por el Pacto Internacional de Derechos Económicos, Sociales y Culturales de 1966 así como por otros documentos de carácter regional-, los derechos sociales constituyen una de las cuestiones centrales de la agenda de todos los partidos y movimientos políticos del mundo occidental. Muy atrás han quedado las voces que a mediados del siglo pasado alertaban de la supuesta incompatibilidad del Estado social con la economía de mercado (Hayek) o con el Estado de derecho (Forsthoff); y, en todo caso, se ha asumido la necesidad de restringir o limitar unos derechos (los derechos de libertad) para proteger otros (los derechos sociales). Lo que hoy se discute no es si deben o no reconocerse y protegerse estos derechos, sino cómo hacerlo sin poner en riesgo la viabilidad económica del Estado. La pregunta acerca del status constitucional de los derechos sociales será incompleta si se interesa únicamente por los aspectos filosóficos o políticos de los derechos. ¿Cuántas constituciones, muy prolijas en el reconocimiento de derechos de todo tipo, carecen de un grado de

\footnotetext{
2 Bobbio 1991: 61-2.
} 
eficacia relevante y suficiente para garantizar las condiciones de vida que se infieren del texto? La articulación de mecanismos jurídicos de garantía y la disponibilidad de medios materiales para hacerlos eficaces son condiciones de posibilidad de la realización de los derechos.

En este trabajo voy a mantener dos tesis, que, si bien son conceptualmente independientes, guardan una estrecha relación. En primer lugar, que cualquier aproximación a los problemas jurídicos de los derechos en general y de los derechos sociales en particular debe tomar como punto de partida la estructura deóntica de los derechos. Su calificación como derechos liberales, derechos políticos o derechos sociales, o como derechos de primera, segunda o tercera generación, inspirada esencialmente por el contexto en el que surgen, es de un indiscutible interés histórico y filosófico ${ }^{3}$, pero secundaria desde el punto de vista jurídico. Tener un derecho, al margen de cuál pueda ser su fundamento filosófico, significa ocupar una posición determinada en el marco de una relación jurídica; afirmar que se tiene derecho a algo sin poder identificar quién está afectado como obligado a hacer o a abstenerse de hacer para lograr su satisfacción es una declaración política o moral; de naturaleza retórica en todo caso.

En segundo lugar, sostendré que la garantía y protección de los derechos sociales está sujeta a límites que deben ser considerados en la determinación de su status constitucional, o más bien, en la configuración de su estructura.

\section{UNA TEORÍA ESTRUCTURAL DE LOS DERECHOS FUNDAMENTALES}

\section{Enunciados sobre derechos}

Los enunciados acerca de los derechos pueden ser, fundamentalmente, de tres tipos. En primer lugar enunciados que afirman las razones que justifican la atribución de un derecho. Este tipo de enunciados se refieren a la fundamentación de los derechos. Un ejemplo de los mismos es el siguiente:

(1) «El derecho a defenderse de la agresión de otros es inherente a la propia naturaleza humana».

Este tipo de enunciados son centrales en las teorías iusnaturalistas de los derechos. Igualmente, son propios de la teoría del interés (Ihering) o de las teorías de los derechos basados en las necesidades humanas. En definitiva, de cualquier teoría de los derechos que centre su atención en el fundamento de la titularidad del derecho.

\footnotetext{
${ }^{3}$ Cfr. Peces-Barba 1995.
} 
Un segundo tipo de enunciados acerca de los derechos son aquellos que indican la posición que un sujeto $a$ ocupa en una relación jurídica; por ejemplo:

(2) « $a$ tiene un derecho a $X »$.

Siendo $a$ el titular del derecho y $X$ el objeto de mismo. Ahora bien, este enunciado está incompleto en tanto no se indique en qué posición queda el otro sujeto de la relación jurídica, el sujeto $b$, tal que:

(2') « $a$ tiene frente a $b$ un derecho a $X »$.

Este tipo de enunciados refleja la dimensión relacional de los derechos, puesto que (2') es equivalente al siguiente enunciado:

$(2$ ') «b tiene frente a $a$ la obligación de $\mathrm{X} »$.

Las implicaciones lógicas entre las posiciones que cada sujeto ocupa en el marco de una relación jurídica fue señalada por W. N. Hohfeld. Según Hohfeld, hay ocho relaciones jurídicas básicas: «derecho», «obligación», «no derecho», «privilegio», «poder», «sujeción», «incapacidad»e «inmunidad» ${ }^{4}$. Cada una de estas posiciones tiene una posición correlativa; así, si $a$ tiene un derecho a $X$ frente a $b, b$ está obligado a $X$ frente a $a$. «Derecho» y «obligación» son posiciones correlativas. Igualmente, si $a$ no tiene un derecho a $X$ frente a $b, b$ no está obligado a $X$ frente a $a$, etc.

Un tercer tipo de enunciados acerca de los derechos es aquél que se refiere a la exigibilidad jurídica y protección de los derechos. Por ejemplo:

(3) «a puede exigir de los Tribunales la satisfacción (por parte de $b$ ) de su derecho a $X »$.

Este tipo de enunciados conecta directamente con aquella teoría de los derechos según la cual «tener un derecho» significa, en sentido estricto, estar habilitado por el orden jurídico a reclamar en los Tribunales el cumplimiento (o, en su caso, una compensación) por parte de un tercero de una obligación no satisfecha ${ }^{5}$. Un derecho que no está garantizado por el ordenamiento jurídico no es un derecho en sentido estricto. Al igual que los enunciados del segundo tipo, éstos enunciados expresan una posición del sujeto $a$ en el marco de una relación jurídica.

Siguiendo esta senda marcada por Kelsen, los enunciados del segundo tipo -(2), (2') y (2' ') - habrían de considerarse superfluos, dado que están presupuestos en los enunciados del tercer tipo; sin embargo, los enunciados del segundo tipo son centrales para la construcción de una teoría estructural de los derechos fundamentales.

\footnotetext{
${ }^{4}$ Hofeld 1913: 30.

${ }^{5}$ Kelsen 1960: 147.
} 
Existen al menos dos razones para no renunciar a los enunciados del segundo tipo ${ }^{6}$. Primero, y aunque un enunciado como (3) no se sigue lógicamente del enunciado (2), este último puede ser visto como una forma simplificada de afirmar (3), incluso cuando la exigibilidad judicial constituye el núcleo de significado del concepto de derecho subjetivo. Segundo, la capacidad jurídica de exigir su cumplimiento forma parte del derecho, pero no parece que agote su contenido. Si así fuera, todos los derechos se reducirían a uno: el derecho a la tutela judicial efectiva. En un sentido lógico, el derecho a $X$ precede a la capacidad jurídica de exigir $X$ : es el fundamento de esa capacidad.

\section{Las posiciones jurídicas}

Una teoría estructural de los derechos fundamentales responde a la estructura deóntica de los derechos, no a cuestiones de fundamentación (al menos, no en este punto) ni a las circunstancias históricas en que han surgido y evolucionado los derechos. Seguiré en este punto las categorías formuladas por Robert Alexy en su Teoría de los derechos fundamentales. Alexy distingue tres posiciones jurídicas básicas que configuran el contenido de los derechos fundamentales: derechos a algo; libertades y competencias ${ }^{7}$.

\subsection{Derechos a algo}

La forma básica de esta posición está expresada en el enunciado (2') «a tiene frente a $b$ (el Estado u otro particular) un derecho a $X »$, donde $X$ puede ser una acción (acción positiva) o una abstención (acción negativa).

a) Derechos a acciones negativas o derechos de defensa. Son derechos de este tipo:

1. Los derechos a que el Estado no interfiera o impida la realización de acciones, bien por imposibilidad fáctica (impidiendo el movimiento, privando de un bien, etc.), bien por imposibilidad jurídica cuando la acción en cuestión es un acto jurídico (privando de las normas que hacen posible la existencia de este tipo de acciones institucionales, como el contrato, el matrimonio, etc.);

2. Los derechos a la no afectación de propiedades personales (vivir, estar sano) o situaciones (inviolabilidad del domicilio) del titular.

3. Los derechos a la no eliminación de posiciones jurídicas, ya sean subjetivas (por ejemplo, derechos subjetivos de propiedad) u

\footnotetext{
${ }^{6}$ Alexy 1997: 181.

7 Alexy 1997: 186 y ss.
} 
objetivas (normas que regulan la institución, por ejemplo, las normas que regulan la adquisición o enajenación de bienes); en otras palabras, una posición de este tipo significa el derecho del ciudadano a que el Estado no derogue determinadas normas.

b) Derecho a acciones positivas o derechos a prestación. El objeto de los derechos a una acción positiva puede ser una acción fáctica (derechos a prestaciones en sentido estricto) o una acción normativa (derechos a prestaciones en sentido amplio). En los derechos a una acción positiva fáctica, el objeto hace referencia al estado de cosas que debe alcanzarse mediante la acción del Estado (por ejemplo, la satisfacción de necesidades básicas de alimento, vestido o techo de cualquier persona). La forma jurídica a través de la cual se alcance es irrelevante a estos efectos. El derecho a una acción positiva normativa tiene por objeto los actos necesarios para la creación de normas estatales. Este tipo de derechos tiene un carácter instrumental o auxiliar; son derechos a la existencia de aquellas normas que permiten la realización de lo que podríamos llamar el núcleo de significado de un derecho fundamental (por ejemplo, el derecho a las medidas organizativas estatales necesarias para la protección del ámbito de libertad que hace posible la libertad de ciencia).

Los llamados derechos sociales (asistencia social, trabajo, educación, etc.) suelen configurarse como derechos a acciones positivas del Estado, pero ni son los únicos que pueden incorporar a su contenido este tipo de posiciones jurídicas, ni la estructura de estos derechos se agota en las prestaciones estatales. Alexy divide los derechos a acciones positivas del Estado en cuatro tipos ${ }^{8}$ :

1. Derechos a protección. El titular de un derecho de protección puede exigir al Estado protección frente a intervenciones de terceros. El objeto de protección puede ser desde la misma vida o la salud, hasta la dignidad, la libertad o la propiedad. La protección puede consistir en medidas jurídicas o en actuaciones directas del aparato estatal.

La diferencia entre los derechos de defensa y estos derechos de protección es que los primeros prohíben cualquier acción que dañe o imposibilite el disfrute del derecho, mientras que los segundos obligan únicamente a realizar alguna de las medidas que garantice el derecho. Los derechos a protección son normas de fin que dejan al legislador la elección del medio para su satisfacción.

\footnotetext{
8 Alexy 1997: 435 y ss.
} 
En relación con estos derechos se plantea la cuestión de si ésta es una categoría autónoma, pues no parece posible pensar en un derecho (que pretenda ser algo más que una mera declaración retórica) que no requiera alguna forma de protección ${ }^{9}$. Lo que esto significa es que forma parte del contenido de todo derecho, fundamental o no, un derecho de protección, además de otras posiciones jurídicas.

2. Derechos a organización y procedimiento. Algo similar ocurre con los derechos a organización y procedimiento; todo derecho fundamental implica posiciones jurídicas de este tipo. No es una cuestión pacífica en la doctrina el que estos derechos se conciban como derechos subjetivos o como garantías objetivas. Estos derechos pueden ser de cuatro tipos:

(i) Competencias de derecho privado. Derechos frente al Estado para que éste formule las normas necesarias (en tanto que constitutivas) para la creación, modificación y extinción de posiciones jurídicas de derecho privado.

(ii) Procedimientos judiciales y administrativos. Este tipo de derechos son una concreción del derecho a una tutela judicial efectiva. Ahora bien, la exigencia constitucional de existencia de estos procedimientos ni agota ni sustituye la exigencia constitucional a una protección efectiva; estos procedimientos aumentan la probabilidad de dicha protección y sus resultados gozan de una presunción derrotable de corrección.

(iii) Organización en sentido estricto. Supone un derecho a la existencia de normas que regulan y propician la cooperación de una número más o menos amplio de personas para alcanzar un mismo fin.

(iv) Formación de la voluntad estatal. Se trata del derecho a que el Estado establezca mecanismos legales de participación política, siendo el más importante, pero no el único, le derecho a voto.

3. Derechos a prestaciones en sentido estricto. Alexy los define como derechos que el individuo tiene frente al Estado a obtener bienes o servicios que, si el individuo tuviera medios financieros suficientes y existiera una oferta suficiente en el mercado, podría obtener de particulares.

\footnotetext{
${ }^{9}$ Hierro 2007: 207.
} 
Algunos de estos derechos a prestaciones están explícitamente reconocidos en los textos constitucionales, otros se obtienen por vía interpretativa. En todo caso, la protección de los derechos sociales puede tener distinta intensidad, según se consideren las normas como (i) vinculantes o programáticas (esto es, exigibles o no ante un tribunal); (ii) derechos subjetivos u obligaciones objetivas del Estado; y (iii) derechos definitivos o derechos prima facie. Estos criterios dan lugar a ocho $\left(2^{3}\right)$ combinaciones posibles, que representan cada uno de ellos un grado distinto de protección ${ }^{10}$.

\begin{tabular}{|c|c|c|c|}
\hline & Derecho vinculante & Derecho subjetivo & Derecho definitivo \\
\hline 1 & + & + & + \\
\hline 2 & + & + & - \\
\hline 3 & + & - & + \\
\hline 4 & + & - & - \\
\hline 5 & - & + & + \\
\hline 6 & - & + & + \\
\hline 7 & - & - & - \\
\hline 8 & - & - & + \\
\hline
\end{tabular}

El grado más alto de protección de un derecho a prestación lo otorgan normas vinculantes que garantizan un derecho subjetivo definitivo, mientras que el grado menor está dado por aquellas normas de carácter político o programáticas que establecen un mero deber objetivo prima facie del Estado a otorgar prestaciones. Cuál sea el grado de protección que corresponda a cada derecho fundamental es algo que depende de la importancia del bien protegido por el mismo. Cuanto mayor es la importancia de ese derecho, más razones existen para que su reconocimiento y protección no se ven sometidas al principio de la mayoría.

\subsection{Libertades}

Entendida como posición en una relación jurídica, la libertad es libertad jurídica. La forma básica de expresar una libertad jurídica es mediante la noción de permiso. Un sujeto $a$ es libre respecto de (la conducta) $X$ cuando está permitido hacer $X$ y está permitido no hacer $X^{11}$.

\footnotetext{
10 Alexy 1997:484.

11 Alexy 1997: 218.
} 
Bajo el concepto de «libertad jurídica» pueden agruparse, no obstante, diversas posiciones jurídicas, por lo que debemos distinguir entre libertades no protegidas y libertades protegidas.

a) Libertades no protegidas: Las libertades no protegidas responden a la definición básica formulada más arriba. Coinciden con lo que von Wright denominó «permiso como (mera) tolerancia» ${ }^{12}$. Las libertades no protegidas son una mera conjunción de permisiones de hacer y de no hacer, pero no llevan asociado ningún derecho a no ser obstaculizado en el ejercicio de esa libertad.

Por otra parte, algunas normas de derecho fundamental son normas permisivas, pero son algo más que una mera tolerancia; adoptan, por tanto, la forma de un permiso explícito o permiso «en sentido fuerte» ${ }^{13}$. La función de estas normas es limitar el ámbito de decisión del legislador ordinario, que no puede mandar o prohibir aquello que permite una norma perteneciente al nivel jerárquicamente superior del ordenamiento. Cuando una libertad es algo más que una conjunción de permisos de hacer y no hacer, entonces estamos ante una libertad protegida.

b) Libertades protegidas: La protección de las libertades va más allá del mero mecanismo jerárquico antes descrito. Las libertades pueden estar protegidas bien por un derecho cuyo contenido es igual al contenido de la libertad (protección directa), bien por el «perímetro protector» del que hablaba Hart ${ }^{14}$, que impide que otros interfieran con determinados medios, pero no con otros, en el ejercicio de la libertad (protección indirecta). Por ejemplo, la libertad de empresa implica la libertad de acción para intentar captar clientes. Los comerciantes están protegidos de ciertas interferencias en esa libertad, como el sabotaje de su negocio o la competencia desleal, pero, al mismo tiempo, no pueden impedir que otros intenten con éxito captar a la clientela mediante otros medios, como una mejor oferta, obstaculizando así el ejercicio de esa libertad. Por otra parte, la protección de las libertades puede realizarse mediante una serie de derechos a algo (protección subjetiva), o mediante normas objetivas (protección objetiva).

Toda libertad estatuida por una norma de derecho fundamental es una libertad frente al Estado y está protegida directa y subjetivamente, esto es, por un derecho de igual contenido a que el Estado no impida u obligue al titular a hacer aquello para lo que se es libre. Dicho de otro modo, toda libertad

12 Von Wright 1979: 103 y ss.

13 Idem.

${ }^{14}$ Hart 1982: 171 y ss. 
que deriva de una norma de derecho fundamental es la combinación de un permiso o libertad no protegida y un derecho de defensa.

\subsection{Competencias}

Aquellos que poseen una competencia pueden modificar situaciones jurídicas, bien mediante la creación de normas válidas (precisamente una de las condiciones de validez de las normas jurídicas es que sean producidas por quien tiene capacidad legalmente reconocida para ello), bien mediante la modificación de la posición de los sujetos en una relación jurídica.

Ahora bien, no toda acción que modifica la posición de los sujetos en una relación jurídica constituye el ejercicio de una competencia. Así, una acción delictiva que genere un derecho de compensación a favor de la víctima no responde al uso de una competencia, aunque modifica la situación jurídica ${ }^{15}$. Las acciones que constituyen un ejercicio de una competencia jurídica son las acciones institucionales, esto es, acciones cuyo sentido viene dado no por su realización fáctica, sino por la existencia de reglas constitutivas ${ }^{16}$. Las normas de competencia son reglas jurídicas de naturaleza constitutiva. Contraer matrimonio, firmar un contrato o aceptar una herencia son acciones jurídicas institucionales que no serían posibles de no existir las normas que confieren competencias (naturalmente, podrían darse los hechos de la convivencia, el cumplimiento de promesas o la posesión de los bienes del fallecido por sus familiares, pero ninguna de estas acciones podría describirse como el ejercicio de un derecho).

La noción de competencia es importante para la completa comprensión de la estructura de los derechos fundamentales. De las normas de derecho fundamental derivan competencias de los ciudadanos; o lo que es lo mismo, existen competencias que gozan de un estatus constitucional. Si el legislador derogara dichas normas que confieren competencias en el derecho privado (por ejemplo, la capacidad para enajenar determinado tipo de bienes inmuebles), o lo hiciera sin una justificación constitucional, estaría violando normas de derecho fundamental. Asimismo, algunas normas de derecho fundamental funcionan como normas de competencia negativas, esto es, normas que limitan el alcance de las normas que confieren competencias al Estado.

\section{Los derechos fundamentales como un conjunto de posiciones jurídicas}

Una vez analizadas individualmente las distintas posiciones fundamentales -y aunque algunas de ellas son discutibles desde el punto de vista de la

15 Alexy 1997:230.

16 Sobre este tipo de reglas, vid. Searle 1980: 43. 
dogmática constitucional- vemos que, en la mayoría de los casos, las normas de derecho fundamental contienen un conjunto de posiciones jurídicas ${ }^{17}$ y que algunas de estas posiciones está contenidas en todas ellas, como se aprecia en el análisis de los derechos a prestaciones en sentido amplio, especialmente con los derechos de protección y los derechos a organización y procedimiento. También respecto de las libertades puede observarse esta estructura compleja. Como vimos, una libertad protegida consiste en la vinculación de una libertad no protegida o mera tolerancia y un derecho a no interferencia en el ejercicio de esa libertad. A estas posiciones se puede añadir una competencia para reclamar ante los tribunales en caso de violación. La suma de estas tres posiciones da lugar a un derecho negativo completo. Si el tipo de protección de la libertad es positivo, entonces la libertad va asociada a un derecho a una acción positiva que haga fácticamente posible lo que es jurídicamente posible. Esa acción positiva puede consistir bien en proteger frente a terceros, mediante un sistema de normas e instituciones de tipo sancionador, bien en algún tipo de prestación. Nada impide que un mismo derecho fundamental incorpore a la libertad jurídica un derecho a acciones positivas y un derecho a acciones negativas.

La configuración completa de cada derecho fundamental es el resultado de un proceso complejo. Siguiendo a Böckenförde ${ }^{18}$, podemos decir que la identificación del contenido de los derechos fundamentales, esto es, el conjunto de posiciones jurídicas constitucionalmente protegidas, depende la teoría de los derechos fundamentales guíe nuestra interpretación de las normas de derecho fundamental. Böckenförde distingue cinco teorías de los derechos fundamentales: la teoría liberal, la teoría institucional, la teoría axiológica, la teoría democrático-funcional y la teoría del Estado social. Así, por ejemplo, la interpretación de la libertad de expresión conforme a una teoría liberal de los derechos fundamentales probablemente configure este derecho como la conjunción de una libertad jurídica y un derecho negativo o derecho de defensa, mientras que una interpretación según una teoría democrática de los derechos incorporará al conjunto de posiciones anterior un derecho a acciones positivas por parte del Estado, puesto que la libertad prensa juega un papel esencial en la formación de una opinión pública informada, que, a su vez, es condición necesaria para garantizar la racionalidad del procedimiento democrático. Nada impide que el intérprete constitucional atribuya a una norma de derecho fundamental diversas posiciones jurídicas que respondan a distintas teorías de los derechos fundamentales.

En todo caso, la configuración completa de los derechos fundamentales y su nivel de protección (cuestión que afecta especialmente a los derechos sociales) no es algo que pueda quedar únicamente sujeto a consideraciones morales o políticas. Los problemas de fundamentación no son los únicos $-\mathrm{y}$

\footnotetext{
17 Alexy 1997:240.

18 Böckenförde 1993: 45 y ss..
} 
me atrevería a decir que tampoco los más importantes-problemas con que se enfrentan hoy los derechos humanos.

\section{LOS LÍMITES DE LOS DERECHOS}

No todo aquello que es deseable y puede ser fundamentado filosóficamente es realizable sin más. La articulación jurídica de los derechos y, en particular, de los derechos sociales está sometida a ciertos límites o condiciones que aquí sólo pueden quedar apuntados.

\section{Límites epistémicos}

Según la célebre frase de De Lolme, «el Parlamento británico [podía] hacerlo todo menos convertir a un hombre en mujer o viceversa». De Lolme estaba doblemente equivocado; lo estaba acerca de la capacidad transformadora del Derecho como instrumento de una voluntad irrestricta, y lo estaba acerca de los límites de lo inconcebible. Es un pensamiento falaz el que atribuye a la potestas del Rey, del Parlamento o del Comité Central la capacidad ilimitada para moldear el conjunto de la sociedad conforme a sus designios. Es lo que Renner denominó «decretinismo».

La genealogía de la concepción instrumental del Derecho puede rastrearse al menos a los orígenes del Estado moderno. En la alta Edad Media (s. xi-xiii) se ponen las bases para el nacimiento del Estado, que concentra el poder político y detenta el monopolio de la creación de derecho. Se abandona una concepción del derecho esencialmente consuetudinaria y localista, es decir, un derecho que es el producto de la interacción social y cuyos centros de producción se encuentran descentralizados, para concebir al Derecho como la voluntad del poder político. Este proceso culmina con el concepto de soberanía de Bodino (s. xvi), a partir del cual, el poder político no reconoce ningún otro poder superior.

El proceso se consolida con la expansión del racionalismo a la política. El derecho es ahora voluntas ratione animata; surge con ello una ciencia de la legislación que derivaría con la revolución francesa en lo que se ha dado en llamar racionalismo constructivista ${ }^{19}$. El primer exponente de este nuevo contexto es Montesquieu quien, aun reconociendo los condicionantes que la naturaleza y los rasgos de cada pueblo suponen para la eficacia y obediencia de las leyes, confía en el ingenio humano para superarlos. Sin embargo, el gran avance en la ciencia de la legislación se produce con Jeremy Bentham. El planteamiento de Bentham va más allá del esquema

\footnotetext{
${ }^{19}$ Hayek 1978, Cap. I.
} 
simple de mandato-sanción y desarrolla diversas técnicas, a través de estímulos indirectos, incluso la desregularización, para alcanzar fines y estados de cosas valiosos.

La transformación del Estado liberal en Estado social ha significado la participación directa en la configuración de las condiciones de vida de los ciudadanos. El Estado no se limita ya a intervenir para supervisar la aplicación de las reglas del juego; su presencia en casi todas las esferas de la vida ha consolidado la idea de que el orden social es producto de la acción directa de la política y de su instrumento más eficaz, el Derecho.

Las normas de derecho fundamental y, en particular, las normas que reconocen derechos fundamentales sociales, establecen fines y estados de cosas que han de ser alcanzados por el Estado: satisfacción de necesidades primarias o de subsistencia, protección de la salud, educación, etc. Ahora bien, el mero reconocimiento de derechos a acciones positivas frente al Estado no es suficiente. La consecución de los objetivos del Estado social, en especial aquellos de tipo estructural, entre los que podemos incluir la igualdad de oportunidades o un determinado grado de libertad real, no sólo exige un escenario socio-económico determinado (algo sobre lo que volveré más adelante), sino también una intervención racional-teleológica que pueda anticipar los efectos a largo plazo, no siempre previsibles y en ocasiones contraintuitivos, de las políticas públicas. Es bien conocida la crítica libertaria a los efectos contraproducentes del asistencialismo: debilitamiento de la iniciativa económica, perpetuación de las situaciones de marginalidad, pobreza espiritual y, en fin, la desactivación de los mecanismos de responsabilidad individual ${ }^{20}$. En este sentido, alguien tan poco sospechoso de insensibilidad social como Stuart Mill sostuvo en sus Principios de Economía Política que el problema, delicado e importante, era «cómo prestar la mayor cantidad de ayuda necesitada, con el menor estímulo a confiarse en ella».

\section{Límites económicos}

Se trata aquí del problema de la escasez. La posibilidad de satisfacción de los derechos, y en especial de los derechos sociales, presupone un grado de riqueza que quienes se preocupan únicamente por la fundamentación de los derechos suelen ignorar.

El análisis estructural muestra que no existen diferencias cualitativas entre los llamados derechos de libertad o de participación política, por un lado, y los derechos sociales, por otro; todos los derechos fundamentales

\footnotetext{
20 Vid. Arias Madonado 2011.
} 
incluyen derechos a prestaciones en sentido amplio (órganos legislativos, judiciales y policiales) y, por tanto, tienen un coste económico más o menos elevado $^{21}$. No obstante, los recursos económicos necesarios para garantizar, hoy por hoy, el derecho a la educación o el derecho a la salud, por no hablar de un supuesto derecho (vinculante, subjetivo y definitivo) a la vivienda, son infinitamente superiores de los que hacen falta para garantizar la libertad de culto o el derecho al honor, aunque circunstancias excepcionales, como la amenaza terrorista, pueden aumentar exponencialmente el coste de la seguridad e integridad físicas.

El problema de la escasez se plantea especialmente con la deriva universalista de los derechos sociales ${ }^{22}$. Originariamente, los derechos sociales eran derechos específicos, concebidos como derechos de aquellos cuya situación de menesterosidad no les permitía valerse por sí mismos o les condenaba a padecer una desigualdad de oportunidades insuperable. La expansión de la titularidad de los derechos sociales incluso a quienes por su nivel de renta podrían satisfacerlos en el mercado privado de bienes y servicios conducen a la hipertrofia del gasto público y al riesgo de colapso económico del sistema. Asimismo, y en la medida en que la deuda pública a largo plazo constituya un problema intergeneracional, se abre aquí un debate moral de enormes dimensiones.

Hay quienes cuestionan el argumento de la escasez. Niegan que se trate de un problema de eficacia productiva, sino un problema político; esto es, la escasez no hace más que encubrir una determinada distribución de los recursos que somete los objetivos que persiguen los derechos sociales a la voluntad política ${ }^{23}$. Ciertamente, cualquier consideración acerca de la escasez parte de un sistema de apropiación y una distribución históricamente dados y, por tanto, modificables, pero tampoco puede obviarse el hecho de que no todo sistema de distribución de la propiedad y de los rendimientos del trabajo es igualmente productivo y genera el mismo nivel de riqueza. Los problemas de una distribución justa de los recursos son importantes, pero «es imprescindible evitar que lo que se distribuya sea la escasez» ${ }^{24}$.

\section{Limites conceptuales}

La idea de Derecho remite a la idea de conflicto; y no sólo al conflicto social, que el Derecho viene a resolver o a encauzar, entre aquellos que tienen acceso a los medios para alcanzar los fines que la sociedad fija como

21 Vid. Ruiz Miguel 1994: 659 y ss.

22 Peces-Barba 1999: 63-4, 2007: 96.

23 Vid. López Calera 1994: 266; Monereo Atienza 2006: 19.

24 González Amuchastegui 1994: 279. 
objetivos de una vida exitosa y aquellos que no tienen acceso a estos medios, sino al conflicto entre los distintos valores y principios morales en que se fundamenta el orden jurídico. Este argumento entronca directamente con la tesis del pluralismo axiológico de Isaiah Berlin, según la cual, la realización de ciertos valores supone, en muchas ocasiones el sacrifico de otros. Que esto signifique que los derechos y bienes constitucionales estén enfrentados entre sí es algo más discutible, aunque es frecuente hablar de «sacrificios justificados» de derechos cuando éstos son limitados por otros derechos. Lo que resulta indiscutible a partir del enfoque de las posiciones jurídicas adoptado más arriba es que toda posición en una relación jurídica tiene una posición correlativa, esto es, todo derecho implica una obligación correlativa. Todos los derechos, y especialmente los derechos a recibir una prestación (en sentido estricto) tienen un coste que el Estado, obligado a satisfacer el derecho, repercute indirectamente a terceros, para lo cual debe reducir o limitar derechos de libertad y de propiedad. Es indiferente que esta relación entre los derechos se aborde desde una perspectiva conflictualista, y se hable de limitación de derechos, o desde una perspectiva coherentista, y se hable de delimitación de derechos; lo relevante aquí es que la configuración de cada derecho determina la configuración del resto de derechos y ello tiene implicaciones éticas y políticas.

\section{Limites políticos}

La cuestión de la protección de los derechos sociales presenta una vertiente política que conecta directamente con los límites epistémicos y económicos, planteando problemas de legitimidad. Como vimos, los derechos de protección pueden ser satisfechos mediante diversas acciones o medidas, que pueden presentar un mayor o menor grado de eficacia, una mayor o menor probabilidad de alcanzar el estado de cosas deseable, afectar en mayor o menor medida las probabilidades de éxito de otros objetivos igualmente susceptibles de protección, etc. La toma de decisiones en condiciones de información limitada implica un margen de error inevitable, pero ¿a quién corresponde el pronóstico y la valoración acerca de los medios de protección? El segundo problema tiene aún mayor relevancia y expresa en toda su intensidad la tensión entre el poder constituyente y el poder legislativo. Dado el indudable impacto presupuestario de los derechos a prestaciones en sentido estricto, que constituyen el núcleo de la mayoría de los derechos sociales, ¿a quién corresponde determinar el grado de protección? La tentación de residenciar en sede constitucional las decisiones acerca la protección de los derechos sociales para sustraerlos de las contingencias del juego democrático no sólo plantea problemas de legitimidad política, sino que parece ignorar todo cuanto se ha dicho anteriormente. 


\section{BIBLIOGRAFÍA}

AleXy, R. Teoría de los derechos fundamentales, trad. Ernesto Garzón Valdés, Madrid: Centro de Estudios Constitucionales, 1997.

Arias Maldonado, M. «La utopía de la sociedad sin impuestos», Revista de Occidente núm. 361, 2011: 55-76.

BobBio, N. El tiempo de los derechos, trad. Rafael de Asís Roig, Madrid: Debate, 1991.

BöCKENFÖRDE, E-W. Escritos sobre Derechos Fundamentales, trad. Juan Luis Requejo Pagés e Ignacio Villaverde Menéndez, Baden-Baden: Nomos, 1993.

GonzÁlez Amuchastegui, J. «Derechos humanos y escasez: entre la economía y la moral», en SAuCA, J. M. a (ed.), Problemas actuales de los derechos fundamentales, Madrid: BOE/Universidad Carlos III de Madrid, (1994): 273-284.

Hayer, F. New Studies in Philosophy, Politics, Economics and The History of Ideas, London, Melbourne and Henley: Routledge \& Kegan Paul, 1978.

Hart, H. L. A. Essays on Bentham, Oxford: Clarendon Press, 1982.

HierRo, L. L. «Los derechos económico-sociales y el principio de igualdad en la teoría de los derechos de Robert Alexy», en V.V.A.A. Derechos sociales y ponderación (edición a cargo de Ricardo García Manrique), Madrid: Fundación Coloquio Jurídico Europeo, 2007: 163-222.

Hohfeld, W. N. «Fundamental Legal Conceptions as Applied in Judicial Reasoning», The Yale Journal vol. 23, No. 1, 1913: 16-59.

López Calera, N. «Escasez y cambio de modelos», en SAuCA, J. M. ${ }^{\text {a }}$ (ed.), Problemas actuales de los derechos fundamentales, Madrid: BOE/Universidad Carlos III de Madrid, 1994: 265-271.

Monereo Atienza, C. «Una teoría de los derechos sociales es posible», en PisArello, G. / VAliño, V. (coords.), Herramientas para la defensa de los DESC. Derechos Económicos, Sociales y Culturales, Barcelona: Observatori DESC, 2006: 9-27.

Peces-Barba Martínez, G. Curso de derechos fundamentales. Teoría General, Madrid: BOE/Universidad Carlos III de Madrid, 1995.

«Los derechos económicos sociales y culturales: apunte para su formación histórica y su concepto», en Id. Derechos sociales y positivismo jurídico (Escritos de Filosofía jurídica y política), Madrid: Dykinson / Universidad Carlos III de Madrid, 1999: 7-66.

- «Reflexiones sobre los derechos sociales», en García ManriQue, R. (ed.), Derechos sociales y ponderación, Madrid: Fundación Coloquio Jurídico Europeo, 2007: 85-101.

Ruiz Miguel, A. «Derechos liberales y derechos sociales», Doxa 15-16, 1994: 651-674.

SEARle, J. Actos de habla. Ensayo de filosofía del lenguaje (trad. Luís Valdés Villanueva), Madrid: Cátedra, 1980.

Von Wright, G. H. Norma y acción. Una investigación lógica (trad. Pedro García Ferrero), Madrid: Tecnos, 1979. 